\title{
Utilising Redundant Voltage Levels for Circulating Current Control in Modular Multilevel Converters
}

\author{
Georgios Konstantinou $^{(1)}$, Josep Pou ${ }^{(1)}$, Salvador Ceballos ${ }^{(2)}$, Ricard Picas ${ }^{(3)}$, \\ Jordi Zaragoza $^{(3)}$ and Vassilios G. Agelidis ${ }^{(1)}$ \\ (1) UNSW Australia, Sydney, NSW, 2052, Australia, (2) Tecnalia Energy, Spain, ${ }^{(3)}$ Technical University of Catalonia, Spain \\ email: g.konstantinou@unsw.edu.au, j.pou@unsw.edu.au, salvador.ceballos@tecnalia.com \\ ricard.picas@upc.edu, jordi.zaragoza-bertomeu@upc.edu, vassilios.agelidis@unsw.edu.au
}

\begin{abstract}
Control of the circulating current in the modular multilevel converter (MMC) is one of the major tasks for the proper operation of the converter topology. This paper develops a controller for the circulating current of the MMC that utilises the redundant voltage levels in $2 N+1$ modulated MMCs in order to regulate the current to its reference. The main advantages of the approach are the elimination of control loops that generate the voltages for the control of the circulating current, simple implementation and very fast dynamic performance. The application of the control is also independent of the circulating current reference. The simplicity and effectiveness of the proposed controller is illustrated through simulations and experimental results.
\end{abstract}

Index Terms-Circulating current, Current control, multilevel converters, modular multilevel converter.

\section{INTRODUCTION}

The modular multilevel converter (MMC) [1]-[3], shown in Fig. 1, and the family of multilevel converters based on series connection of sub-modules (SMs) [4] have defined the state of the art in multilevel power electronics conversion over the last decade. They offer an expandable and redundant configuration [5] capable of generating high number of voltage levels and provide high quality voltages and currents with high efficiency and low switching losses [2], [3].

Owing to its many advantages, different configurations of SMs serving multiple functions [6] and the provision of a single dc-link for the overall topology, the MMC is well-suited to grid applications, most prominently high-voltage direct-current (HVDC) power transmission, as well as STATCOMs, traction systems [2] and battery energy storage systems (BESS) [2], [3]. Its application to medium power variable speed drives is also of interest [7], with the low-speed, low fundamental frequency operation posing a particular set of challenges for the converter.

Major tasks for the control and proper operation of the MMC include the control of output quantities (voltages and currents) as well as internal quantities (SM capacitor voltages and circulating currents). Regulating the SM capacitor voltages is imperative in order to provide balanced and symmetrical output while maintaining the ratings and limits of the SMs. On the other hand, circulating currents have a significant effect in the optimal operation of the converter and are mainly driven by the voltage and energy imbalance between the arms of the

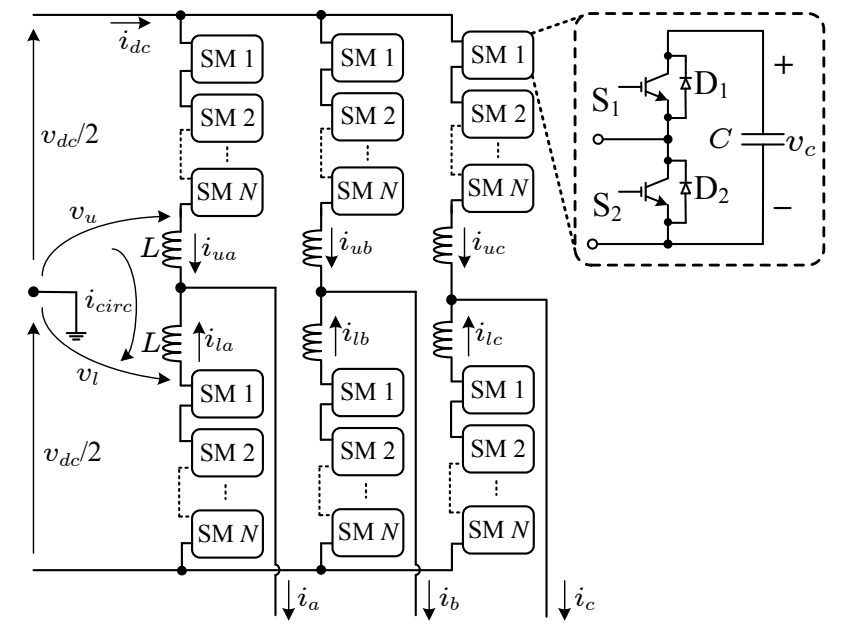

Fig. 1. Circuit configuration of a three-phase MMC.

converter. For both quantities, a set of controllers [8]-[22] is typically employed to ensure that all requirements are met during the operation of the converter.

The objective of this paper is to propose and demonstrate the operation of a controller for the MMC circulating currents that utilizes the pulse-width modulation patterns and, more specifically, the available redundancies in the voltage waveform of an MMC under $2 N+1$ modulation, where $N$ is the number of SMs per arm. The controller acts directly on the circulating current, selecting the state of the SMs within the arm so that the circulating current is driven to its reference value. It eliminates the need for additional voltage reference injections to the output voltage reference of the converter or further control loops.

The work is organized in the following manner. Section II provides an overview of MMC circulating currents and relevant current control methods proposed in the existing technical literature. Section III presents relevant background of $2 N+1$ modulation for the MMC and introduces the circulating current controller based on the redundant switching states. Simulation and experimental results of the proposed controller are given in Section IV and the conclusions of the work are summarized in Section V. 


\section{Circulating Current And Control}

Assuming an infinite number of SMs within the MMC arms $(N \rightarrow \infty)$, the converter can be represented with the equivalent circuit of Fig. 2(a). The value of the variable capacitors $C_{u}$ and $C_{l}$ depends on the number of seriesconnected SMs per arm. Under the assumption of $L \rightarrow 0$, the output current will be distributed amongst the two arms based on the value of these capacitors and, hence, the number of inserted SMs. Under a sinusoidal modulation signal $v_{a m}$ $\left(v_{a m} \in[-1,1]\right)$

$$
v_{a m}=m_{a} \cos (\omega t),
$$

where $m_{a}$ represents the converter modulation index and a phase current $i_{a}$ which are expressed as:

$$
i_{a}=\hat{I}_{a} \cos (\omega t+\phi),
$$

the currents through the upper and lower arms, and consequently through the upper and lower arm capacitors, can be written as [21]:

$i_{u}=\frac{\hat{I}_{a}}{2} \cos (\omega t+\phi)+\frac{m_{a} \hat{I}_{a}}{4} \cos (2 \omega t+\phi)+\frac{m_{a} \hat{I}_{a}}{4} \cos (\phi)$,

and

$i_{l}=\frac{\hat{I}_{a}}{2} \cos (\omega t+\phi)-\frac{m_{a} \hat{I}_{a}}{4} \cos (2 \omega t+\phi)-\frac{m_{a} \hat{I}_{a}}{4} \cos (\phi)$.

where The first term in (3) and (4) is equal to half of the phase current demonstrating that the upper and lower phasearms share the output current $i_{a}$ equally. The second and the third terms in (3) and (4) represent the second order harmonic and the dc component of the circulating current $\left(i_{c i r c}=\frac{i_{u}-i_{l}}{2}\right)$ within the upper and lower phase-arms, respectively. The do component is the only component associated with active power exchange between the dc-side and the ac-side of the MMC.

The instantaneous power at the dc side of the MMC is:

$$
p_{d c}=V_{d c} I_{d c} .
$$

The average power is calculated by:

$$
P_{d c}=\frac{1}{T} \int_{0}^{T} p_{d c} d t=\frac{m_{a} \frac{V_{d c}}{2}}{\sqrt{2}} \frac{\hat{I}_{a}}{\sqrt{2}} \cos (\phi),
$$

or equivalently,

$$
P_{d c}=V_{a} I_{a} \cos (\phi)=P_{a c},
$$

where $V_{a}$ and $I_{a}$ are the rms values of the phase voltage and current, respectively. In the case of a balanced three-phase operation, the extracted current from the dc side of the MMC will be a dc component and the second order harmonic of the circulating currents within the three-phase legs are canceled out.

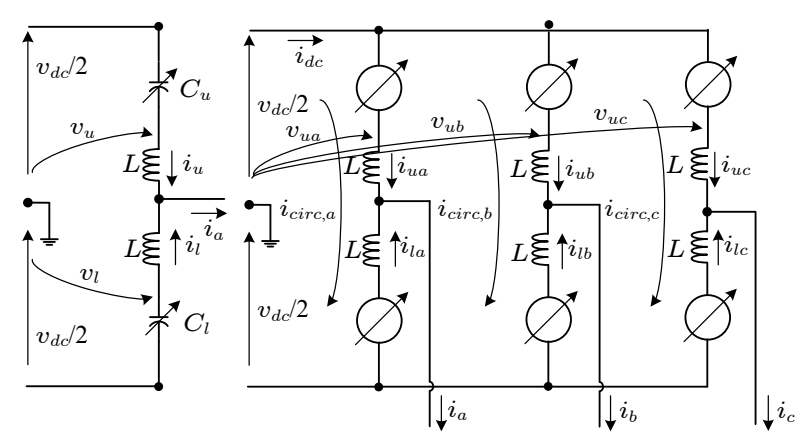

(a)

(b)

Fig. 2. Equivalent circuits of the MMC, (a) assuming infinite number of SMs in the converter arms, (b) three-phase circuit with arms as variable sources.

\section{A. Current Control Methods}

An MMC can operate without a controller for the internal arm currents $\left(i_{u} \& i_{l}\right)$ or the circulating current. Additional benefits can be gained if these currents are regulated to a reference value which can be determined based on various control objectives. The benefits from controlling the circulating current have been identified from the very early stages of research into the MMC [8], [9] and multiple controllers with different control objectives have been proposed [8]-[21].

In order to maintain the energy within the SM capacitors to the required level, hence avoiding a continuous increase or decrease of the SM capacitor voltages and unstable operation, a dc current component is necessary within the circulating current. The value of the dc current can be determined from (5) and (6), and is given by

$$
I_{d c}=\frac{m_{a} \hat{I}_{a}}{4} \cos (\phi) .
$$

The dc value of (8) defines the simplest reference of the circulating current for the MMC $\left(i_{c i r c}=I_{d c}\right)$, in which case the arm currents are expressed as

$$
\begin{gathered}
i_{u}=\frac{1}{2} i_{a}+I_{d c}, \text { and } \\
i_{l}=\frac{1}{2} i_{a}-I_{d c} .
\end{gathered}
$$

To achieve the dc reference, the higher order harmonics and predominantly the negative sequence second order harmonic [8] should be suppressed or eliminated. This can be achieved in the synchronous reference frame (SRF) [8] with the use of linear controllers or through directly filtering the higher order harmonics with the use of low-pass filters (LPF) in the measured circulating current, as shown in Fig. 3(a). A decoupled current controller for the output and circulating current was proposed in [10] in a method that can be extended to other modular topologies such as the Modular Multilevel Matrix Converter of [11]. A circulating current control method based on energy within the arms and controllers on the positive and negative synchronous reference frame was presented in [13] and under unbalanced conditions in [12]. . 


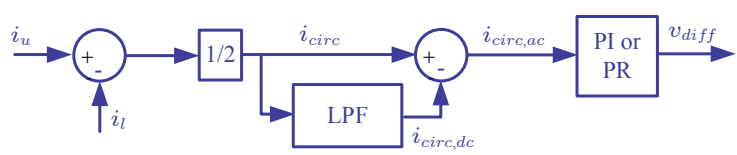

(a)

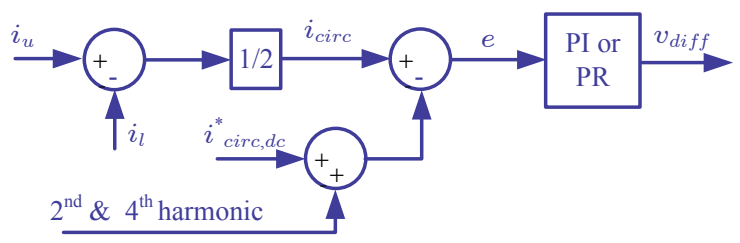

(b)

Fig. 3. Control of the circulating current, (a) Elimination of ac components and, (b) Injection of higher order harmonics

The predominant harmonic that needs to be eliminated from the circulating current is of second order, meaning that proportional or proportional integral controllers might not offer the necessary tracking characteristics. In order to improve the performance of reference tracking, various control methods that include resonant [14]-[16] and repetitive [17], [18] controllers have also been proposed with an example shown in Fig. 3(b). Resonant controllers can be included for higher frequencies (such as the 4th, 6th etc) [14] without affecting the dc component of the circulating current but every additional loop adds to the complexity of the system. The impact of dead time in the performance of PR controllers was presented in [15] where an increase in the \% THD and a deterioration in the system performance was identified. The operation of the MMC under both balanced and unbalanced conditions considering non-ideal PR control, in an effort to reduce the infinite gain of ideal PR controllers at the resonant frequency, was shown in [16].

Elimination of higher order harmonics in the circulating current provides the lowest rms value for the arm current, resulting in the most efficient operation of the converter in terms of switching and conduction losses [8], [21]. However, including higher order harmonics in the circulating current [9], [20] provides secondary benefits to the MMC, predominantly reduction of the capacitor voltage ripple [9], allowing reduction in size of the SM capacitors. The off-line optimisation of [20] calculates the optimal level of 2 nd and 4th order harmonic injection in the circulating current for all operating points and load conditions, while analytical methods [9] can provide similar performance. An issue of these methods is the high computational cost or complexity when more than two harmonics (mainly the second and fourth) are included in the current reference.

In order to simplify the calculation of injection levels, a reference for the circulating current can be derived from values readily available to the current controller [21] as:

$$
i_{\text {circ }}^{*}=\frac{i_{a} v_{a m}}{2},
$$

with the total reference also including actions of PI controllers

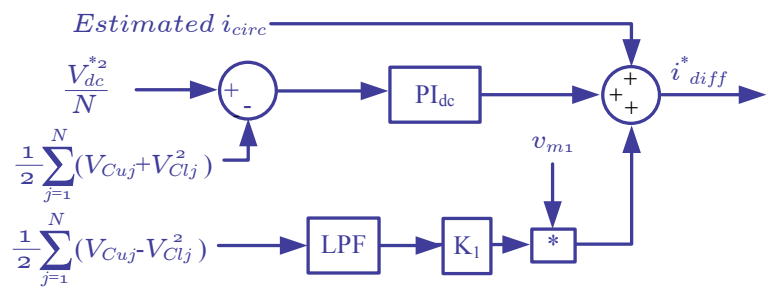

Fig. 4. Generation of circulating current control reference of the MMC using instantaneous measurement values.

to facilitate energy balancing between the converter arms, as shown in Fig. 4. It was shown in [21] that the reference of (11) provides results close to those of the analytically determined optimal injections without introducing significant complexity.

A common point of all previous methods is that they use an additional controller (PI, PR, repetitive, etc) in order to generate a voltage reference $\left(v_{d i f f}^{*}\right)$ for the control of the circulating current within each phase-leg. This voltage reference is then added and subtracted from the phase-leg reference $\left(v_{a m}\right)$ to generate the individual references of the two arms, providing control of the circulating current without affecting the output of the MMC phase-leg.

\section{Proposed Circulating CURRent Controller}

\section{A. $2 N+1$ Modulation for the MMC}

One of the interesting properties of the MMC is that, for a given configuration with $N$ SMs per arm, it can generate $N+1$ or $2 N+1$ voltage levels in the output. The $2 N+1$ can be achieved either through carrier interleaving - phase-shifting the carrier waveforms of the upper and lower arm by $180^{\circ}$ [23] - or by directly modulating the converter with $2 N$ levels (carriers) and properly distributing the switching signals to the upper and lower arm SMs. In both cases, if a sorting algorithm is used to facilitate voltage balancing, the only necessary output is the number of SMs in the upper $\left(n_{u}\right)$ and lower $\left(n_{l}\right)$ arm of the MMC.

The additional voltage levels are intermediate levels to that of $N+1$ modulation (denoted with the $\pm 1 / 2$ in Fig. 5). They are generated when the number of SMs connected to the phaseleg of the converter is equal to either $N+1$ or $N-1$ so that for level $x+1 / 2$ two possible combinations exist, one with $n_{u}=x$ and $n_{l}=N-(x-1)$ and a second with $n_{u}=x+1$ and $n_{l}=N-x$. These redundant states are a result of connecting or removing SMs from either the upper of the lower arm so that $\left(n_{u}+n_{l}\right)$ is the same, while $\left(N-n_{u}-n_{l}\right)$ is \pm 1 .

The two redundant states have opposite effect in the circulating current within the arms despite generating the same voltage level. Considering the differential mode equivalent circuit of the MMC phase-leg, the two states can be described through an added SM with two different polarities, negative, as shown in Fig. 6(a) when $N+1$ and positive when $N-1$ SMs are connected in the phase-leg (Figs. 6(a) and (b) respectively). 


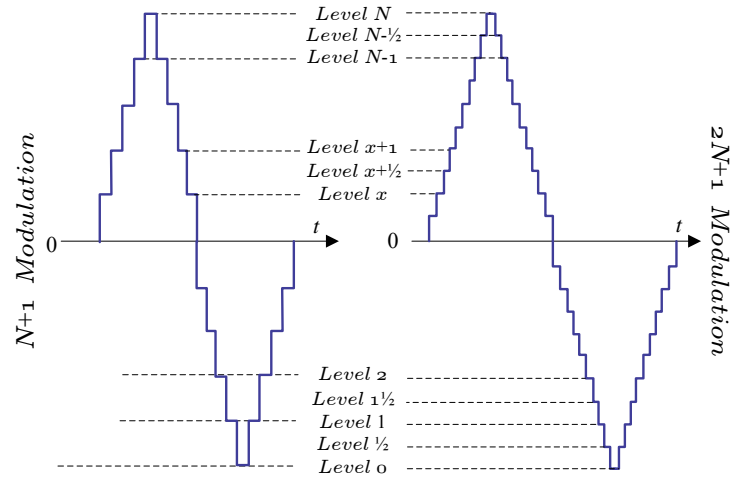

(a)

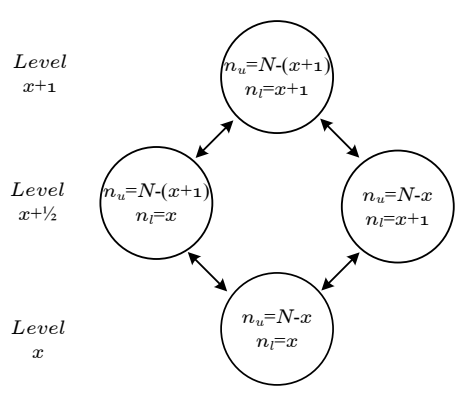

(b)

Fig. 5. (a) Multilevel waveforms of $N+1$ and $2 N+1$ modulated MMCs and (b) transitions between adjacent levels and number of SMs in $2 N+1$ modulated MMCs.

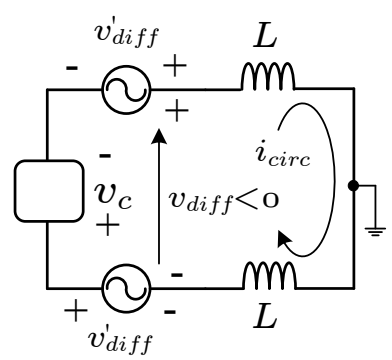

(a)

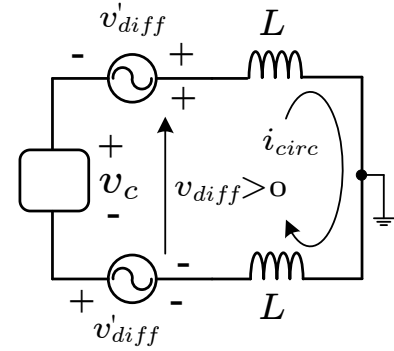

(b)
Fig. 6. Differential mode equivalent circuit, (a) $N+1 \mathrm{SMs}$ in the phase-leg, (b) $N-1 \mathrm{SMs}$ in the phase-leg.

\section{B. Proposed Controller}

Based on the analysis of the previous section and the description of states from Fig. 5(b), we can construct a tree that demonstrates all possible voltage levels and their effect on the circulating current. This is shown in Fig. 7 for five consecutive voltage levels of a multilevel waveform.

The proposed controller makes use of the two redundant states available and selects the one that regulates the circulating current towards the reference. This means that when $i_{\text {circ }}$ is above the reference $\left(i_{\text {circ }}\right)$ then $N+1 \mathrm{SMs}$ are connected to the phase leg and when $i_{c i r c}$ is below the reference, $N-1$ SMs are connected. The only information, therefore, needed to select among the two states is whether $i_{\text {circ }} \geq i_{\text {circ }}^{*}$ or not. The above selection takes place during a transition from an $N R$
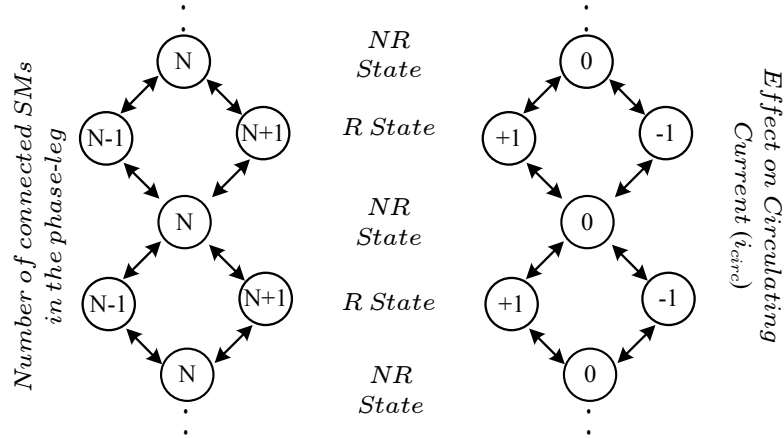

Fig. 7. Redundant switching states amongst consecutive voltage levels and effect on the circulating current

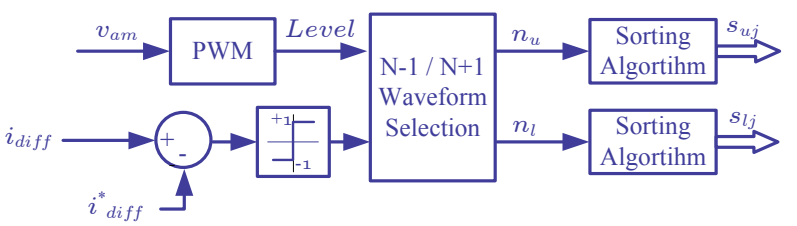

(a)

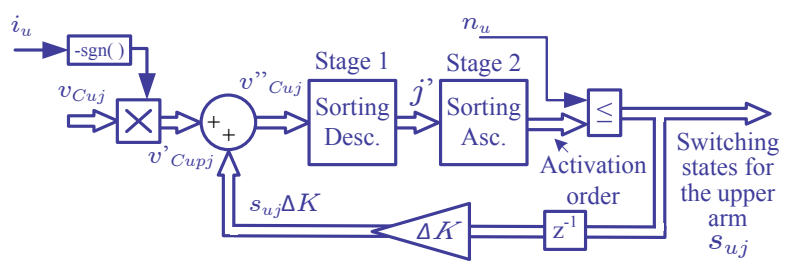

(b)

Fig. 8. MMC circulating current control and modulation, (a) Proposed implementation of circulating current control within a phase-leg, (b) modified voltage sorting and balancing algorithm.

state to an $R$ state (Fig. 7) and can be treated as connecting or bypassing one SM during the given transition. The reference signals for the upper and lower arm $\left(v_{a m u}\right.$ and $\left.v_{a m l}\right)$ remain unaffected and so does the switching frequency of the SMs within the converter arms.

The implementation of the proposed current controller is shown in Fig. 8(a). The reference signal together with the modulator define the level of the multilevel waveform while a simple comparison between the actual and the measured reference current provides the information necessary for the controller to select between the two redundant states. Any of the control methods of Section II can be used as the reference $i_{\text {circ }}^{*}$ with the end result being the number of SMs required in the upper and lower arms of the converter. Voltage balancing without excessive increase in the switching frequency of the converter is achieved through the restricted voltage balancing algorithm of [22], as shown in Fig. 8(b). However, any of the voltage balancing methods proposed in the literature [2], [3] can be applied without any effect to the proposed controller.

\section{Simulation \& ExPERIMENTAL Results}

The operation of the proposed controller is demonstrated through simulations using a single-phase MMC with $N=5$ 
TABLE I

PARAMETERS OF THE Simulation AND EXPERIMENTAL CONVERTERS

\begin{tabular}{ccc}
\hline \hline Parameter & Simulation & Experiment \\
\hline \hline Number of SMs per arm, $N$ & 5 & 5 \\
Dc-link voltage, $V_{d c}$ & $5000 \mathrm{~V}$ & $250 \mathrm{~V}$ \\
SM reference voltage, $V_{C}$ & $1000 \mathrm{~V}$ & $50 \mathrm{~V}$ \\
SM capacitor, $C$ & $3.6 \mathrm{mF}$ & $3.6 \mathrm{mF}$ \\
Arm inductors, $L$ & $3.6 \mathrm{mH}$ & $3.6 \mathrm{mH}$ \\
Load $R_{L} \& L_{L}$ & $22 \Omega \& 5 \mathrm{mH}$ & $15.6 \Omega \& 5 \mathrm{mH}$ \\
Carrier frequency $f_{\text {car }}$ & $5 \mathrm{kHz}$ & $2 \mathrm{kHz}$ \\
\hline \hline
\end{tabular}

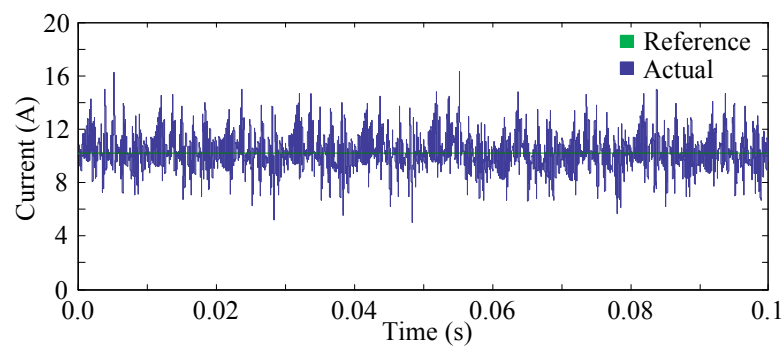

(a)

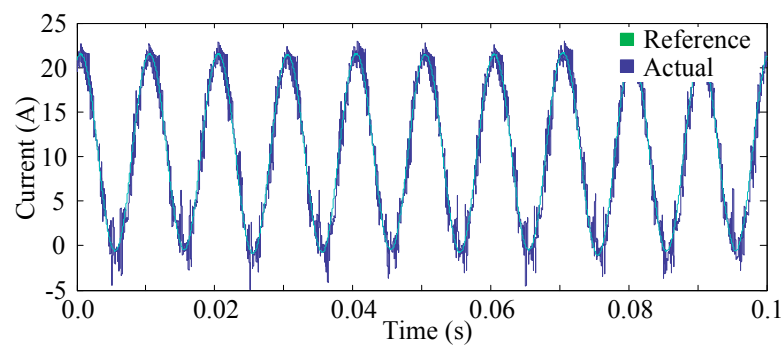

(b)

Fig. 9. Simulation results of the proposed controller when the circulating current is (a) a dc component and, (b) a dc and ac component.

SMs per arm of the converter capable of generating 11 voltage levels in the output voltage waveform. The specifications of the simulated system and the single-phase experimental prototype are given in Table I.

Two different circulating current control references are used for the control of the current, including the dc current reference of (8) and that of (11) [21]. The simulation results of the $i_{\text {circ }}$ together with the corresponding references are shown in Figs. 9(a) and (b), respectively. In both cases, the controller is capable of regulating the circulating current around its reference. As the proposed controller does not affect the actual voltage levels but only the selection of the switching states, the current ripple that is present in the current waveform due to the $2 N+1$ modulation is still present, as also shown in Fig. 9 .

The operation and performance of the proposed controller can also be analysed from the perspective of higher order harmonics in the circulating current. When a current controller is not implemented, the harmonic content of the circulating current is only influenced from the modulation of the upper and the lower arm and includes a significant harmonic at

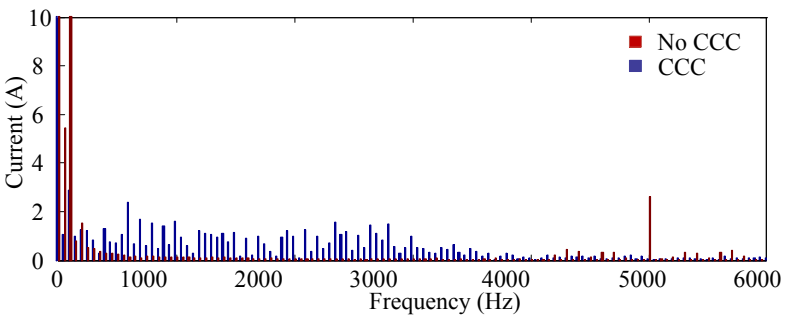

Fig. 10. Harmonic spectra of the circulating current under $2 N+1$ modulation with and without the proposed circulating current controller.

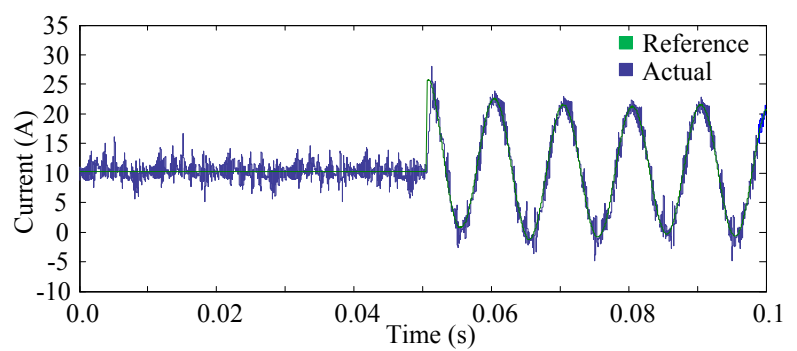

(a)

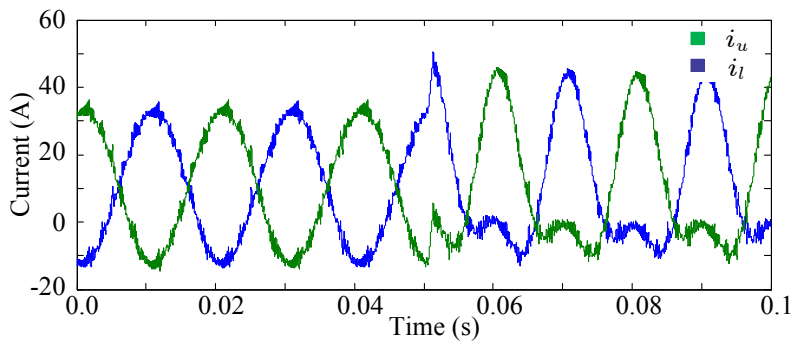

(b)

Fig. 11. Transition between the two circulating current references (a) circulating current, (b) arm currents.

the equivalent switching frequency together with the second order harmonic. This harmonic is the switching frequency component of the upper and lower arms that is not cancelled out int he circulating current due to the interleaved operation of the converter. The implementation of the proposed circulating current controller spreads the harmonic content of $i_{\text {circ }}$ as it tries to regulate it towards its reference, affecting the regular pattern and the previously present higher order harmonics. These can be observed through an FFT of the circulating current, with and without current control, in Fig. 10.

To demonstrate the dynamic performance of the proposed controller, a change between the two circulating current references, from a dc circulating current to an ac, is introduced in Fig. 11. The controller is capable of following the reference and tracking the transition instantaneously. In both cases, the SM capacitor voltages are well regulated to the reference value as shown in Fig. 12. The reduction in the SM capacitor voltages, as a result of the harmonics injected in the circulating current [21], is also demonstrated.

Fig. 13 shows the measured upper and lower arm currents 


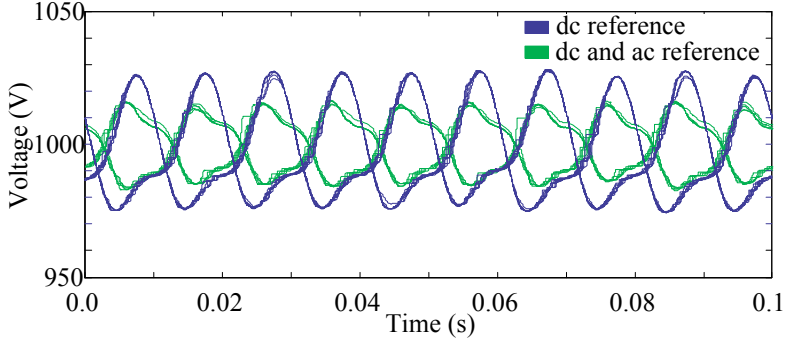

Fig. 12. Simulated SM capacitor voltages with the proposed circulating current control under dc and dc\&ac circulating currents.

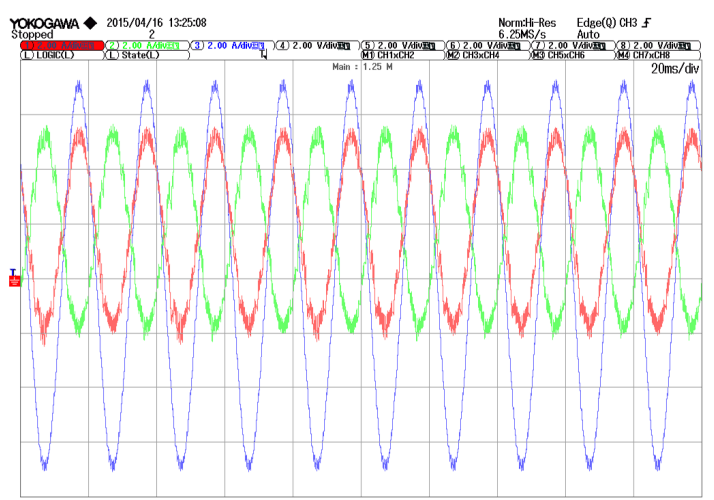

Fig. 13. Experimental results with dc circulating current reference $\mathrm{CH} 1$ : upper arm current $i_{u} ; \mathrm{CH} 2$ : lower arm current $i_{l}, \mathrm{CH} 3$ : load current $i_{a} ; \mathrm{CH} 1, \mathrm{CH} 2$ CH3: $2 \mathrm{~A} / \mathrm{div}$.

$\left(i_{u}\right.$ and $\left.i_{l}\right)$ in the arms of the experimental prototype under a dc circulating current reference. The results are in agreement with the arm currents under dc circulating current reference of Fig. 11(b). The harmonics within the circulating current do not appear in the output current $\left(i_{a}\right)$.

\section{CONCLUSION}

In this paper, a controller for the circulating current of the MMC based on the redundant states of $2 N+1$ modulation has been proposed. The two redundant states have opposite effect to the circulating current and the controller uses the deviation from the reference (either positive or negative) to select amongst them. The controller is implemented in the modulation stage without affecting the converter switching frequency, does not require injections to the reference waveform of the converter and is independent of the circulating current reference. Simulation and experimental results with two different current control methods verify the operation and demonstrate the performance of the proposed controller.

\section{REFERENCES}

[1] A. Lesnicar and R. Marquardt, "An innovative modular multilevel converter topology suitable for a wide power range," in Proc. IEEE Bologna PowerTech Conference, 23-26 Jun. 2003, Bologna, Italy.

[2] M. A. Perez, S. Bernet, J. Rodriguez, S. Kouro, and R. Lizana, "Circuit topologies, modelling, control schemes and applications of modular multilevel converters," IEEE Trans. Power Electron., vol. 30, no. 1, pp. 4 - 17, Jan. 2015
[3] S. Debnath, J. Qin, B. Bahrani, M. Saeedifard, and P. Barbosa, "Operation, control, and applications of the MMC: A review," IEEE Trans. Power Electron., vol. 30, no. 1, pp. 37 - 53, Jan. 2015.

[4] H. Akagi, "Classification, terminology, and application of the modular multilevel cascade converter (MMCC)," IEEE Trans. Power Electron., vol. 26, no.11, pp. 3119-3130, Nov. 2011.

[5] G. Konstantinou, J. Pou, S. Ceballos, and V.G. Agelidis, "Active redundant sub-module configuration in modular multilevel converters," IEEE Trans. on Power Delivery, vol. 28, no. 4, pp. 2333-2341, Oct. 2013

[6] G. Konstantinou, J. Zhang, S. Ceballos, J. Pou and V.G. Agelidis, "A comparative review of sub-modules for modular multilevel converters," Proc. IEEE PEDS 2015, June 2015, Sydney, Australia, pp. 1-6.

[7] A. Antonopoulos, L. Angquist, S. Norrga, K. Ilves, L. Harnefors, and H. Nee, "Modular multilevel converter AC motor drives with constant torque from zero to nominal speed," IEEE Trans. Ind. Applic., vol. 50, no.3, pp. 1982-1993, May-Jun. 2014.

[8] Q. Tu, Z. Xu, and L. Xu, "Reduced switching-frequency modulation and circulating current suppression for modular multilevel converters," IEEE Trans. on Power Delivery, vol. 26, no.3, pp. 2009-2017, Jul. 2011.

[9] S.P. Engel, and R.W. de Doncker, "Control of the modular multilevel converter for minimised cell capacitance," in Proc. European Conf. on Power Electronics and Applications (EPE), Aug. 2011, Birmingham, UK

[10] R. Lizana, M.A. Perez, D. Arancibia, J.R. Espinoza, and J. Rodriguez "Decoupled Current Model and Control of Modular Multilevel Converters," IEEE Trans. Ind. Electron., vol.62, no.9, pp.5382-5392, Sept. 2015.

[11] F. Kammerer, J. Kolb, and M. Braun, "Fully decoupled current control and energy balancing of the Modular Multilevel Matrix Converter," Power Electronics and Motion Control Conference (EPE/PEMC), 4-6 Sept. 2012, pp. 1-8

[12] M. Ji-Woo, K. Chun-Sung, P. Jung-Woo, K. Dea-Wook, and K. JangMok, "Circulating current control in MMC under the unbalanced voltage," IEEE Trans. Power Del., vol. 28, no. 3, pp. 1952-1959, Jul. 2013.

[13] G. Bergna, E. Berne, P. Egrot, P. Lefranc, A. Arzande, et al., "An energybased controller for HVDC modular multilevel converter in decoupled double synchronous reference frame for voltage oscillation reduction,' IEEE Trans. Ind. Electron., vol. 60, no.6, pp. 2360-2371, Jun. 2013.

[14] L. Zixin, W. Ping, C. Zunfang, Z. Haibin, L. Yongjie, and L. Yaohua, "An inner current suppressing method for modular multilevel converters,' IEEE Trans. Power Electron., vol. 28, no. 11, pp. 4873-4879, Nov. 2013.

[15] B. Chen, Y. Chen, C. Tian, J. Yuan, and X. Yao, "Analysis and suppression of circulating harmonic currents in a modular multilevel converter considering the impact of dead time," IEEE Trans. Power Electron., vol. 30, no. 7, pp. 3542-3552, Jul. 2015.

[16] S. Li, X. Wang, Z. Yao, T. Li, and Z. Peng, "Circulating current suppressing strategy for MMC-HVDC based on non-ideal proportional resonant controllers under unbalanced grid conditions," IEEE Trans. Power Electron., vol. 30, no.1, pp. 387-397, Jan. 2015.

[17] H. Liqun, Z. Kai, X. Jian, and F. Shengfang, "A repetitive control scheme for harmonic suppression of circulating current in modular multilevel converters," IEEE Trans. Power Electron., vol. 30, no. 1, pp. 471-481, Jan. 2015.

[18] Z. Ming, H. Long, Y. Wenxi, and L. Zhengyu, "Circulating harmonic current elimination of a CPS-PWM-based modular multilevel converter with a plug-in repetitive controller," IEEE Trans. Power Electron., vol. 29, no. 4, pp. 2083-2097, Apr. 2014.

[19] J. Jung, H. Lee, and S. Sul, "Control strategy for improved dynamic performance of variable-speed drives with modular multilevel converter,' IEEE J. Emerg. Sel. Topics Power Electron., vol. PP, pp. 1-1, 2014, doi: 10.1109/JESTPE.2014.2323955

[20] R. Picas, J. Pou, S. Ceballos, J. Zaragoza, G. Konstantinou, and V.G. Agelidis, "Optimal injection of harmonics in circulating currents of modular multilevel converters for capacitor voltage ripple minimization," in Proc. IEEE ECCE Asia 2013, Melbourne, Australia.

[21] J. Pou, S. Ceballos, G. Konstantinou, V. G. Agelidis, R. Picas, and J. Zaragoza, "Circulating current injection methods based on instantaneous information for the modular multilevel converter," IEEE Trans. on Ind. Electr., vol. 62, no. 2, pp. 777-788, Feb. 2015.

[22] R. Darus, J. Pou, G. Konstantinou, S. Ceballos, V.G. Agelidis and R. Picas, "A modified voltage balancing algorithm for the modular multilevel converter: evaluation for staircase and phase-disposition PWM', IEEE Trans. Power Electron., vol. 30, no. 8, pp. 4119-4127, pp. 1-8.

[23] K. Ilves, L. Harnefors, S. Norrga, and H. Nee, "Analysis and operation of modular multilevel converters with phase-shifted carrier PWM," IEEE Trans. Power Electron., vol. 30, no. 1, Jan. 2015, pp. 268-283. 